\title{
QUANTUM OPTICS OF ATOMIC WAVE-PACKETS
}

\author{
K. RZĄżEWSKI \\ Centrum Fizyki Teoretycznej PAN
}

Al. Lotników 32/46, 02-668 Warszawa, Poland

L. Plaja-Rustein, L. Pumares-Carceller, L. Roso-Franco

Departamento de Fisica, Universitat Autonoma de Barcelona, 08193, Bellaterra, Spain

AND W. ŻAKOWICZ

Institute of Physics, Polish Academy of Sciences

Al. Lotników 32/46, 02-668 Warszawa, Poland

\begin{abstract}
Recent progress in production and control of the atomic wave-packets calls for re-examination of the most fundamental quantum optical processes. We have examined the evolution of the atomic wave-packet resonantly driven by the monochromatic laser beam and the spontaneous emission from an extended atom. We stress the role of Doppler shifts in the dephasing of the atomic dipole. New effects of single laser pulse photon echo and homogeneous Doppler modification of the emission line are discussed.
\end{abstract}

PACS numbers: 42.50.Vk, 42.50.Md

\section{Introduction}

Laser cooling techniques have led to the preparation of very slow and coherent atomic beams capable of displaying wave properties of atoms. The atomic version of the cclcbrated Young interference expcriment using atomic de Broglie waves has become possible $[1,2]$. Earlier similar experiments with individual massive particles, such as electrons or neutrons, have also been performed. An important difference comes from the rich internal structure of atoms. Moreover, the interaction with light offers a remarkably effective tool for probing this internal structure. It allows for the interplay between the internal and external degrees of freedom.

Until now the resonant interaction of atoms with light was typically studied either completely neglecting the coupling between radiative transitions and the center of mass motion (see for instance [3]) or, as in the theory of laser cooling, including the coupling only under the assumption of an incoherent density matrix for the atomic motion. 
In this lecture we look again at two fundamental processes discussed in quantum optics: resonant driving of a single atomic transition (so-called Rabi oscillations) and (the most fundamental QED process) spontaneous emission. We look for the modification of these processes due to the coherence of the center of mass wave function.

\section{Rabi oscillations of atomic wave-packet}

The interaction of an atom with a monochromatic laser beam will be discussed in the two-dimensional geometry of a continuous-wave Gaussian atomic beam. The atoms travel in the direction $z$ and the transverse direction of the beam is $x$. The suitable solution of the time dependent Schrödinger equation for the free atom may be expanded into the plane wave momentum eigenfunctions in the following way:

$$
\begin{aligned}
& \Psi(x, z, t)=\exp (-\mathrm{i} E t) \\
& \times \int \mathrm{d} p \mathrm{~d} q \delta\left(E-\frac{p^{2}}{2 M}-\frac{q^{2}}{2 M}\right) \exp [\mathrm{i}(q x+p z)] \exp \left[-\frac{(a q)^{2}}{2}\right],
\end{aligned}
$$

where $a$ is the width of the beam at its waist $(z=0)$ and $E$ is the encrgy of the atoms in the beam. Of course the Gaussian character of the beam is easily recovered if, as we assume, most of the energy is in the forward direction $(1 / a)^{2} / 2 M \ll E$.

Now, the atomic beam encounters a monochromatic light beam crossing in $x$-direction. The photon frequency is assumed to be exactly resonant with some atomic transition. We denote the light wave's frequency and wave vector by $\omega$ and $k$. Geometry of the interacting beams is shown in Fig. 1 . The Hamiltonian of

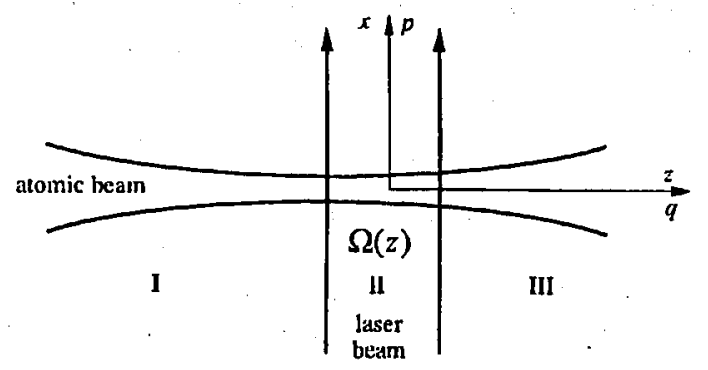

Fig. 1. Scheme of the coherent, Gaussian atomic beam crossing the $\mathrm{cw}$ laser beam, resonant with one of the dipole transitions of the hydrogen atom.

the total system (external and internal degrees of freedom) in the rotating wave approximation has the form

$$
H_{\mathrm{RWA}}^{\prime}=\frac{p^{2}+q^{2}}{2 M}+\hbar \omega \sigma_{3}+\Omega(z)\left\{\sigma_{-} \exp [\mathrm{i}(\omega t-k x)]+\text { h.c. }\right\} .
$$

Passing to a rotating frame allows us to get rid of the explicit time dependence in the Hamiltonian'(2.2). The resulting time independent IIamiltonian reads

$$
H_{\mathrm{RWA}}=\frac{p^{2}+q^{2}}{2 M}+\Omega(z)\left[\sigma_{-} \exp (-\mathrm{i} k x)+\text { h.c. }\right] \text {. }
$$


Now we can solve the time independent Schrödinger equation

$$
I I_{\mathrm{RWA}} \Psi_{E}=E \Psi_{E}
$$

first assuming a two-dimensional incident plane wave of atoms in their lower state $(-)$. If we disregard the $z$-dependence of the laser intensity $(\Omega(z)$ constant inside the laser beam) the problem resembles the scattering on a square well potential. As in that case, we can write the solution of (2.4) separately in regions I, II, III, (recall Fig. 1) and then determine the unknown coefficients through the matching conditions. The wave function in three regions takes the form

$$
\begin{aligned}
& {\left[\begin{array}{c}
\psi_{+}^{\text {I }} \\
\psi_{-}^{\text {I }}
\end{array}\right]=\left[\begin{array}{c}
r_{+} \exp \left(-\mathrm{i} q^{\prime} z\right) \exp [\mathrm{i}(p+k) x] \\
{\left[\exp (\mathrm{i} q z)+r_{-} \exp (-\mathrm{i} q z)\right] \exp (\mathrm{i} p x)}
\end{array}\right],} \\
& {\left[\begin{array}{c}
\psi_{+}^{\text {II }} \\
\psi_{-}^{\text {II }}
\end{array}\right]=\left[\begin{array}{c}
\sum_{j=1,2, \mp} d_{j, \mp} \exp \left[\mp \mathrm{i} q_{j} z+\mathrm{i}(p+k) x\right] \\
\sum_{j=1,2, \mp} f_{j, \mp} \exp \left(\mp \mathrm{i} q_{j} z+\mathrm{i} p x\right)
\end{array}\right],} \\
& {\left[\begin{array}{c}
\psi_{+}^{\text {III }} \\
\psi_{-}^{\text {II }}
\end{array}\right]=\left[\begin{array}{c}
t_{+} \exp \left(\mathrm{i}^{\prime} z\right) \exp [\mathrm{i}(p+k) x] \\
t_{-} \exp [\mathrm{i}(q z+p x)]
\end{array}\right] .}
\end{aligned}
$$

Of course the $x$-component of the momentum of the atom in the upper level $(+)$ is increased by the momentum of one absorbed photon. The reflected and transmitted upper level atoms have a modified $z$-component of momentum according to the conservation of energy

$$
E=\frac{q^{2}+p^{2}}{2 M}=\frac{q^{\prime^{2}}+(p+k)^{2}}{2 M} .
$$

Inside the laser field the atomic wave travels in the form of a superposition of two dressed states with longitudinal momenta

$$
\frac{q_{1,2}^{2}}{2 M}=\frac{q^{2}}{2 M}+\frac{\Delta}{2} \pm \sqrt{\Omega_{0}^{2}+\frac{\Delta^{2}}{4}}
$$

where the detuning $\Delta$ is equal to

$$
\Delta=\frac{p^{2}}{2 M}-\frac{(p+k)^{2}}{2 M} \text {. }
$$

In the limit of a very weak excitation, $\left(\Omega_{0} \ll \Delta\right)$, the dressed states travel with the longitudinal momenta $q$ and $q^{\prime}$ and are almost purely lower and upper levels. IIence, the transverse momenta are, like in a field free regions, $p$ and $p+k$, respectively. In the other limit of very strong driving, both dressed states have practically equal mixture of the lower and upper states. They nevertheless travel with different longitudinal velocities. For one of the dressed states the laser beam acts like a potential well while for the other it is a potential barrier. With an equal amount of the lower and upper levels, the dressed states travel in the transverse direction with the average of the lower and the upper level momenta, so they both travel with the momentum $(p+p+k) / 2=p+k / 2$.

Of course in the intermediate situation when the Rabi frequency is comparable to the detuning, we have two dressed states, the one with stronger population in the lower level drifts in the transverse direction with a momentum a bit higher 

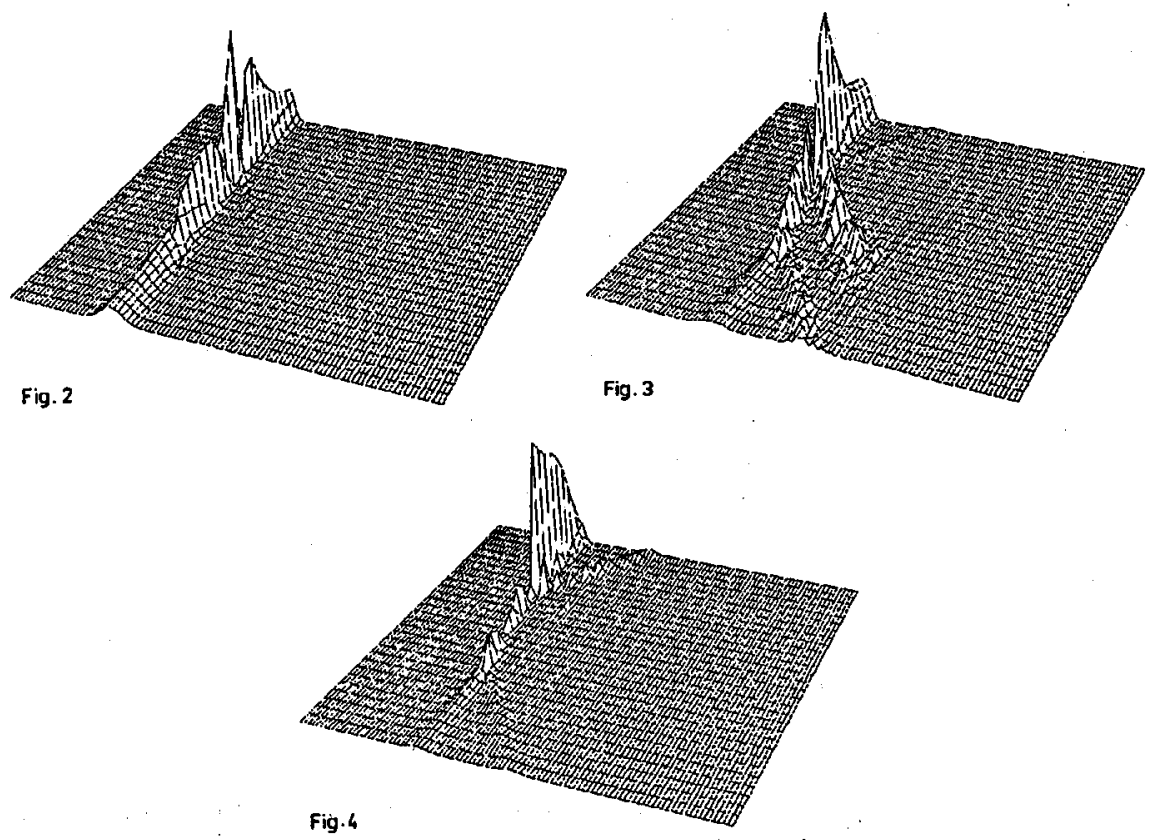

Fig. 2. Atomic beam of Fig. 1 undergoing Rabi oscillations and splittings discussed in our paper. $\Omega_{0}=0.08 \times 10^{-9}$ a.u. Weak driving. Dressed states almost coincide with the bare states of the atom.

Fig. 3. The same as Fig. $2, \Omega_{0}=2 \times 10^{-9}$ a.u. Intermediate driving. Dressed states almost equally populated.

Fig. 4. The same as Fig. 2, $\Omega_{0}=10^{-8}$ a.u. Strong driving. Dressed states drift with momentum $k / 2$ and do not split. Note strong reflection.

than $p$ and the one closer to the upper level travels with a momentum somewhat below $p+k$.

The general expression for the wave function inside the laser beam contains also reflected waves.

We illustrate now the splitting in Figs. 2-4. In all figures the Gaussian atomic beam enters from behind. Its parameters are

$$
\begin{array}{ll}
\text { mass of the atom (hydrogen): } & M=1850 \text { a.u., } \\
\text { width of the transverse momentum distribution: } & \frac{1}{a}=10^{-4} \text { a.u., } \\
\text { total kinetic energy of atoms: } & E=2.7 \times 10^{-6} \text { a.u.. }
\end{array}
$$

The atomic beam crosses the laser beam which comes from the left. The wave vector of the light is 10 times larger than the spread of the transverse momenta: $k=10^{-3}$ a.u., so that the absorption of one photon is enough to kick the atom out of its initial transverse momentum distribution. This is required to see the individual dressed states inside the laser beam. The width of the laser beam is $d=0.025 \mathrm{~cm}$.

The three figures differ by the value of the Rabi frequency. It is equal to 
$\Omega_{0}=0.08 \times 10^{-9}$ a.u. in Fig. $2,2 \times 10^{-9}$ a.u. in Fig. 3 , and $10^{-8}$ a.u. in Fig. 4. It illustrates all three regimes discussed above. Of course the content of upper and lower states of each dressed state becomes visible outside the laser beam. Note a strong reflected signal of excited atoms in Fig. 4.

The results show that the interaction with the laser beam may modify the shape of the atomic wave-packets in the transverse direction. In particular, the lower (or upper) level atoms can display a multipeak splitting as a result of the interaction with a single laser beam.

\section{Single laser beam photon echo}

According to classical electrodynamics the atomic dipole radiates if it is oscillating with a nonzero amplitude. Quantum electrodynamics adds the possibility of the spontaneous cmission: The quantum mechanical expectation value of the dipole moment may be zero, but the expectation of its square (inversion) is different than zero and this is a source of the incoherent light. The coherent, semiclassical part of the radiation is due to the external driving by the resonant laser light. But also at the exit of the light beam the atoms may have a nonzero dipole moment and would still radiate classically. In the case of the collection of atoms moving with different velocities the dephasing of the dipole moment due to the Doppler shifts leads to a terminating fluorescence signal known as a free induction decay. The dephasing may be reversed by the interaction with another laser beam. It leads to another burst of fluorescence radiation known as a photon echo [4].

Something analogous may happen in the coherent atomic beam interacting with the single laser beam considered in this paper. In this case the cohercnt part of the radiated field is driven by the expectation value of the spatial density of the dipole moment rather than the dipole moment itself. But this density is simply proportional to a product of lower and upper level wave functions

$$
d(x, z) \approx d \operatorname{Re}\left[\psi_{+}^{*}(x, z) \psi_{-}(x, z)\right] .
$$

In Fig. 5 we show a somewhat idealized sketch of the interacting beams. Of course

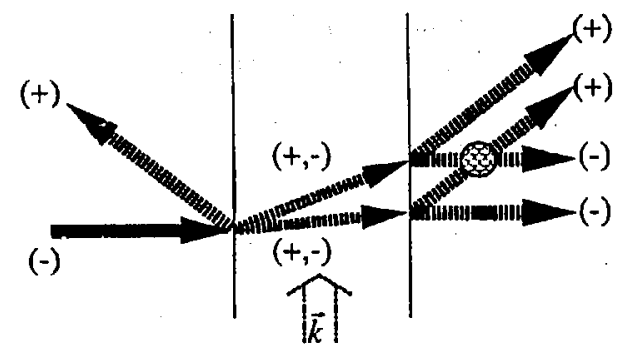

Fig. 5. Sketch of a single laser beam photon echo. Non-vanishing value of the density of dipole moment, a source of coherent radiation, is nonzero also outside of the laser beam. 
the density of the dipole moment is nonzero also in the region where lower level component of the stronger deflected dressed state crosses the upper level component of the less deflected dressed state. This is another example of an effect caused by the combination of atomic beam coherence, coherence of the laser-atom interaction and the recoil due to absorption and emission of photons. We call this new phenomenon "a one laser pulse photon echo".

Clearly radiation of the strongly driven atomic beam requires further study.

\section{Spontaneous emission from an extended wave-packet}

The most obvious omission of the model studied so far is the neglect of spontaneous emission. We are going to correct this partially by reviewing here our recent paper devoted to the problem of spontaneous emission from an extended wave-packet [5].

Several mechanisms of modification of spontaneous emission have been discussed and also demonstrated experimentally. Among these modifications the most important is the inhibition and the enhancement of spontaneous emission due to the modification of the vacuum modes in a resonant cavity [6,7]. A natural question of possible modification of the spontaneous emission due to the coherence of the center of mass motion has been addressed only recently $[5,8]$.

To study this problem we consider the simplest IIamiltonian

$$
H=\frac{p^{2}}{2 M}+\frac{\hbar \omega_{0}}{2} \sigma_{z}+h \sum_{j} \omega_{j} a_{j}^{+} a_{j}+h \sum_{j}\left[\lambda_{j} a_{j}^{+} \sigma_{-} \exp \left(-\mathrm{i} k_{j} \cdot r\right)+\text { h.c. }\right],
$$

where $r$ and $p$ are the center of mass position and momentum of the atom, $a_{j}$ and $a_{j}^{+}$are the annihilation and creation operators of the electromagnetic field modes, and $\sigma_{i}$, the Pauli matrices, act on the internal degree of freedom of the atom.

The state vector may be expressed in terms of the atom and photon state vectors

$$
|\psi(t)\rangle=\int \mathrm{d}^{3} p \alpha(p, t)|p,+, 0\rangle+\sum_{j} \int \mathrm{d}^{3} p \beta_{j}(p, t)\left|p-\hbar k_{j},-, \boldsymbol{k}_{j}\right\rangle,
$$

where the arguments of the kets denote: the atomic momentum, the internal state $(+$ or - ) of the atom and the momentum of the outgoing photon. The amplitude equations resulting from the Schrödinger equation read

$$
\begin{aligned}
& \dot{\alpha}(p, t)=-\mathrm{i}\left(\frac{p^{2}}{2 M}+\frac{\omega_{0}}{2}\right) \alpha(p, t)-\mathrm{i} \sum_{j} \lambda_{j} \beta_{j}(p, t), \\
& \dot{\beta}_{j}(p, t)=-\mathrm{i}\left[\frac{\left(p-k_{j}\right)^{2}}{2 M}-\frac{\omega_{0}}{2}+\omega_{j}\right] \beta_{j}(p, t)-\mathrm{i} \lambda_{j} \alpha(p, t),
\end{aligned}
$$

where we have omitted Planck's constant. Equations (4.3) and (4.4) should be solved with the initial condition $\alpha(p, 0)=\alpha_{0}(p)$ and $\beta_{j}(p, 0)=0$.

The equations are easily solved in the Laplace transform domain. Under standard single pole approximation, including the frequency shift in the level spacing, the solutions are

$$
\alpha(p, t)=\alpha_{0}(p) \exp \left(-z_{0} t\right)
$$




$$
\beta_{j}(p, t)=-\mathrm{i} \lambda_{j} \alpha_{0}(p) \frac{\exp \left(-z_{0} t\right)-\exp \left(-z_{j} t\right)}{z_{0}-z_{j}},
$$

where

$$
z_{0}=\mathrm{i}\left(\frac{p^{2}}{2 M}+\frac{\omega_{0}}{2}\right)+\gamma_{0}
$$

and

$$
z_{j}=\mathrm{i}\left[\frac{\left(p-k_{j}\right)^{2}}{2 M}-\frac{\omega_{0}}{2}+\omega_{j}\right] .
$$

The spontaneous emission width is given by the standard expression

$$
\gamma_{0}=\pi \sum_{j}\left|\lambda_{j}\right|^{2} \delta\left(\omega_{0}-\omega_{j}\right)
$$

It is easy to see that the extended atom decays the same way as the point atom

$$
P(t)=\int \mathrm{d}^{3} p|\alpha(p, t)|^{2}=\exp \left(-2 \gamma_{0} t\right) \int \mathrm{d}^{3} p\left|\alpha_{0}(p)\right|^{2}=\exp \left(-2 \gamma_{0} \ell\right)
$$

but the properties of the spontaneously emitted light are modified. Indecd, for $t \rightarrow \infty$ the probability $P_{j}$ of emitting the photon of wave vector $k_{j}$ is

$$
\begin{aligned}
& P_{j}=\int \mathrm{d}^{3} p\left|\beta_{j}(p, t)\right|^{2}= \\
& \quad\left|\lambda_{j}\right|^{2} \int \mathrm{d}^{3} p\left|\alpha_{0}(p)\right|^{2} \frac{1}{\left[\omega_{0}-\omega_{j}+p \cdot k_{j} / M+k_{j}^{2} / 2 M\right]^{2}+\gamma_{0}^{2}},
\end{aligned}
$$

which may be interpreted as the result of independent emission from each momentum component of the wave function. Each momentum contribution is simply a Lorentzian line centered around the Doppler shifted position. Upon integration over the frequencies of the photons emitted in the given direction we obtain the angular distribution of the light. Neglecting the small recoil energy in the denominator, we may easily perform the resulting Lorentzian integral obtaining

$$
I(\hat{n})=I_{0}(\hat{n}) \int \mathrm{d}^{3} p \frac{\left|\alpha_{0}(p)\right|^{2}}{1-p \cdot \hat{n} / M c},
$$

where the angular distribution $I_{0}(\hat{n})$ describes the standard dipole pattern. Hence, as long as the initial wave-packet does not contain relativistic velocity components, the total intensity is not affected by the wave-packet. This finding is in disagreement with the implications in Ref. [8].

An interesting modification arises in the spontaneous emission spectrum. Since the initial momentum distribution can be quite complicated, besides the broadening substantial distortions of the spectrum are possible.

As an example let us consider an initial wave-packet which has two peaks in the coordinate space at the distance $d$. Its Fourier transform would have a periodic modulation with the period in the momentum space given by $2 \pi / d$. A two-peak Gaussian wave-packet with components of width $a$ separated by $d$ has a momentum distribution

$$
\left|\alpha_{0}(p)\right|^{2}=\frac{a^{3}}{(2 \pi)^{3 / 2}} \frac{1}{1+\exp \left(-d^{2} / 2 a^{2}\right)} \exp \left(-\frac{1}{2} p^{2} a^{2}\right)[1+\cos (p \cdot d)] .
$$




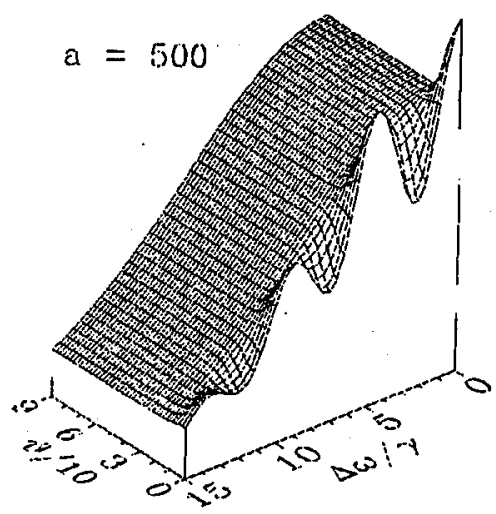

Fig. 6. Angular distribution of the emission spectrum from a two-peak Gaussian wave-packet. The width of each peak $a=500$ a.u., the distance between the peaks $d=10000$ a.u. The angle is measured from the direction of $d$. We have assumed a typical radiative transition in the hydrogen atom.

In Fig. 6 we show the spectrum as a function of the angle $\theta$ between the vector $\boldsymbol{d}$ and the direction of observation. We note the oscillations and their fast suppression with growing $\theta$. Of course, this oscillations would be missing if not for the mutual coherence of the two peaks of the wave function.

\section{Acknowledgments}

The authors would like to thank J.H. Eberly and N. Bigelow for interesting discussions. K.R. and L.P. enjoyed their hospitality at the Dept. of Physics and Astronomy of the University of Rochester where a part of this work was done.

\section{References}

[1] O. Carnal, J. Mlynek, Phys. Rev. Lett. 66, 2689 (1991).

[2] D.W. Keith, C.R. Ekstrom, Q.A. Turchette, D.E. Pritchard, Phys. Rev. Lett. 66, $2692(1991)$.

[3] L. Allen, J.H. Eberly, Optical Resonance and Two-Level Atoms, Dover, New York 1987.

[4] N.A. Kurnit, I.D. Abella, S.R. Hartmann, Phys. Rev. Lett. 13, 567 (1964).

[5] K. Rzążewski, W. Żakowicz, J. Phys. B 25, L319 (1992).

[6] D. Kleppner, Phys. Rev. Lett. 47, 233 (1981).

[7] S. Haroche, J.M. Raimond, Advances in Atomic and Molecular Physics, Vol. 20, Eds. D. Bates, B. Bederson, Academic Press, New York 1985.

[8] J. Robert, Ch. Miniatura, O. Garceix, S. Le Biteux, V. Lorent, J. Reinhardt, J. Boudon, J. Physique II 2, 601 (1992). 